\title{
Erratum to: Spinocerebellar Ataxia Type 6: Molecular Mechanisms and Calcium Channel Genetics
}

\author{
Xiaofei Du and Christopher Manuel Gomez
}

\section{Erratum to:}

Chapter 7 in: C. Nóbrega and L. Pereira de Almeida (eds.), Polyglutamine Disorders, Advances in Experimental Medicine and Biology 1049, https://doi.org/10.1007/978-3-319-71779-1_7

In the original version of the book, the term "spinocerebellum ataxia" has been changed to read as "spinocerebellar ataxia" in Chapter 7. The erratum chapter and the book have been updated with the change. 